\title{
Culture and Differentiation of Monocyte Derived Macrophages Using Human Serum: An Optimized Method
}

\author{
Marie Saghaeian-Jazi, ${ }^{1}$ Saeed Mohammadi, ${ }^{2,}$ and Sima Sedighi ${ }^{3}$ \\ ${ }^{1}$ Student Research Committee, Golestan University of Medical Sciences, Gorgan, IR Iran \\ ${ }^{2}$ Department of Molecular Medicine, Faculty of Advanced Medical Technologies, Golestan University of Medical Sciences, Gorgan, IR Iran \\ ${ }^{3}$ Bones, Joints and Connective Tissue Research Center, Golestan University of Medical Sciences, Gorgan, IR Iran \\ "Corresponding author: Saeed Mohammadi, Department of Molecular Medicine, Faculty of Advanced Medical Technologies, Golestan University of Medical Sciences, Gorgan, \\ IR Iran. E-mail: s.mohammadi@goums.ac.ir
}

Received 2015 January 08; Accepted 2015 March 11.

\begin{abstract}
Background: Monocyte derived macrophages (MDMs) are appropriate in vitro models to study the function of macrophages in immune related diseases. Not only most of the methods in literature for efficient MDM culture and differentiation are expensive but also they require specific equipment. However, there are some reports indicating that monocyte enrichment is possible through attachment. Purification and differentiation of macrophages occurs in media containing human serum or platelet depleted plasma without extra supplementations. Different variables such as incubation time and serum concentration affect this simple MDM preparation method.

Objectives: Here we represent an optimized simple method for MDM preparation from peripheral blood mononuclear cells (PBMC). Materials and Methods: To introduce an optimized method we accomplished the present descriptive study. After PBMC isolation and monocyte enrichment in complete RPMI 1640 growth media, macrophages were cultured and differentiated using human serum. Efficient phagocytosis was evaluated using heat-inactivated Escherichia coli followed by SYBR staining. Geimsa staining of macrophages was accomplished to visualize the typical morphology under light microscopy.

Results: The derived macrophages have the typical morphology of differentiated macrophages and are able to phagocyte the heat inactivated SYBR stained E. coli.

Conclusions: This optimized method is a simple and cost effective method to prepare MDM with typical morphology representations able to phagocytosis efficiently.
\end{abstract}

Keywords: Macrophage, Monocyte, Differentiation, Optimization

\section{Background}

Macrophages are professional scavenger immune cells which regulate innate and adaptive immunity responses $[1,2]$. Interestingly, the proper function of macrophages is related to immune regulation in some infectious, autoimmune and inflammatory diseases [2]. To study the function of macrophages in vitro, the monocyte derived macrophage (MDM) is an acceptable and cost effective model [3-5]. Current protocols for MDM preparation require expensive process of monocyte purification by magnetic cell sorting (MCS) and needs colony stimulating factors such as granulocyte colony stimulating factor (GCSF) and granulocyte macrophage colony stimulating factor (GMCSF) for macrophage differentiation [6, 7]. However, there are some more simple and economic methods for MDM culture and differentiation [8-11].

\section{Objectives}

Here, we optimized an attachment mediated monocyte enrichment method following by the differentiation of macrophages in human derived serum [11] to obtain functional macrophages capable of phagocytosis.

\section{Materials and Methods}

We conducted the present descriptive study to introduce a novel and modified method for culturing and also obtaining functional macrophages using peripheral blood mononuclear cells (PBMC). The optimized procedure includes the following steps.

\subsection{Peripheral Blood Mononuclear Cells (PBMC) Isolation}

A volume of $10 \mathrm{~mL}$ venous blood was collected in a sterile tube containing $1.1 \mathrm{~mL}$ sodium citrate $3.8 \%$ as an anticoagulant [8] and centrifuged for 25 minutes at $3000 \mathrm{rpm}$. 
The buffy coat layer was separated and diluted in $4 \mathrm{~mL}$ sterile phosphate buffered saline (PBS) $\left(37^{\circ} \mathrm{C}\right)$. The diluted buffy coat was overlaid on Ficoll-Paque (1: 1 ratio) followed by centrifugation in $2500 \mathrm{rpm}$ for 10 minutes. The peripheral blood mono nuclear cells were precisely separated and washed with $\mathrm{PBS}\left(37^{\circ} \mathrm{C}\right) 3-5$ times to eliminate any red blood cell (RBC) contamination.

\subsection{Monocyte Enrichment}

PBMCs were suspended in 5 mL RPMI 1640 (Invitrogen) supplemented with $1 \%$ penicillin-streptomycin (Invitrogen) and $0.5 \%$ human serum or platelet depleted plasma and incubated in original, gelatin $2 \%$ coated or plasma treated T25 tissue culture flasks in a $5 \% \mathrm{CO}_{2}$, humidified, $37^{\circ} \mathrm{C}$ incubator for 3 hours. Then, the floating cells consisting of lymphocytes and remaining RBCs were removed by aspiration and attached cells were washed vigorously by PBS for at least 3 times. This wash step is critical for the purity of monocyte separation [9].

For gelatin coating, $3 \mathrm{~mL}$ sterile gelatin solution $2 \%$ in PBS was added to each T25 flasks and was fully aspirated after 2 hours of incubation in $37^{\circ} \mathrm{C}$. The autologous plasma was centrifuged in $16000 \mathrm{~g}$ in $4^{\circ} \mathrm{C}$, then was used to treat flasks for $30 \mathrm{~min}$ in $37^{\circ} \mathrm{C}$. Plasma treated flasks were used for monocyte enrichment after aspiration and PBS washing. Plasma treatment provides a fibronectin covered surface, which enhances monocyte attachment [12].

\subsection{Macrophage Culture and Differentiation}

The monocytes were cultured in RPMI 1640 (Invitrogen) supplemented with $1 \%$ penicillin-streptomycin (Invitrogen) and $10 \%$ human $\mathrm{AB}$ positive serum or $10 \%$ FBS (Invitrogen) in a $5 \% \mathrm{CO}_{2}$, humidified, $37^{\circ} \mathrm{C}$ incubator up to 2 weeks. The culture media were renewed every 3 days and cells monitored morphologically for differentiation.

\subsection{E. coli Heat Inactivation, SYBR Staining and Phagocytosis}

Heat inactivated E. coli $\left(95^{\circ} \mathrm{C}\right.$ for 1 hour) were suspended in TE (Tris-EDTA) buffer $(108 / \mathrm{mL})$ and were stained using 0.1\% SYBR safe stain (SinaClon BioScience) for 30 minutes in dark. Following by 3 PBS wash steps (5 minutes, $10000 \mathrm{rpm}$ ), SYBR stained heat inactivated E. coli were suspended in RPMI 1640 and added to macrophages to a final concentration of $107 / \mathrm{mL}$ E. coli for a population of about 106 macrophages. Efficient phagocytosis was allowed by incubation in $37^{\circ} \mathrm{C}$ for 1 hour and avoiding light. The macrophages were then washed 3 times with $37^{\circ} \mathrm{C}$ PBS and fixed in paraformaldehyde $4 \%$ for 10 minutes. The nucleus was stained with $1 \mu \mathrm{g} / \mathrm{mL}$ DAPI (4', 6-diamidino-2phenylindole) and fluorescent microscopy was employed for imaging the cells.

\subsection{Geimsa Staining of Macrophages}

Simultaneously, 108/mL suspended heat inactivated $E$. coli in TE buffer were resuspended in RPMI 1640 and added to macrophages to a final concentration of $107 / \mathrm{mL}$ E. coli for a population of about 106 macrophages. Efficient phagocytosis was allowed by incubation in $37^{\circ} \mathrm{C}$ for 1 hour. The macrophages were then washed 3 times with $37^{\circ} \mathrm{C}$ PBS. Macrophages were then fixed by adding 100\% methanol to the rinsed wells. After 1 minute, a fresh solution of $10 \%$ Geimsa stain (Geimsa's azur-eosin-methylene blue, Merck Millipore) in distilled water was used to stain macrophages for 20 minutes. Finally the Geimsa stained wells would be rinsed off using distilled water and macrophages would be visualized using light microscopy (HPF: 400x).

\section{Results}

Peripheral blood mono nuclear cells consist of a heterogeneous population of mononuclear cells including lymphocytes, natural killer cells and monocytes [13]. Monocytes distinguishable ability for attachment makes it possible to purify them from PBMC simply $[14,15]$. Different variables could affect the efficiency of purification [5, $8,9,11,14,16]$. To achieve the most favorable attachment condition for monocytes, we tested non gelatin-treated, gelatin $(2 \%)$ coated, plasma treated or both gelatin and plasma treated tissue culture plates. Approximately, $2.5 \times$ 106 PBMCs were settled down in RPMI 1640 (0.5\% AB positive human serum, $1 \%$ penicillin-streptomycin) in $5 \% \mathrm{CO}_{2}$ incubator for 3 hour. Lymphocytes were aspirated afterwards and monocytes were washed to remove all the remaining floated or loosed cells. Finally, monocytes were visualized by light microscopy and then detached to count by the use of Trypan blue $0.4 \%$ and hemocytometer. A total number of $1.5 \times 106$ monocytes were acquired from a $10 \mathrm{~mL}$ blood volume $(1.5 \times 105$ per blood $\mathrm{mL})$. As shown in Figure 1 the percentage of monocyte to PBMCs is about $5 \%$ which is in normal range. There were no obvious differences of enriched monocyte populations in different plates coated with gelatin, plasma or gelatin and plasma together as attachment enhancers with monocytes enriched in nontreated tissue culture plates (not shown).

Attachment triggers the differentiation of monocytes to macrophages [14] and some cytokines like granulocyte and granulocyte-monocyte colony stimulation factors (GCSf, GMCSF) enhance this process [17]. There are some evidences indicating FBS or human serum can alternatively supply the necessary cytokines and colony stimulation factors (CSFs) for the maturation of macrophages [8,11].

Here, we compared the efficiency of differentiation and viability of macrophages in media supplemented with 


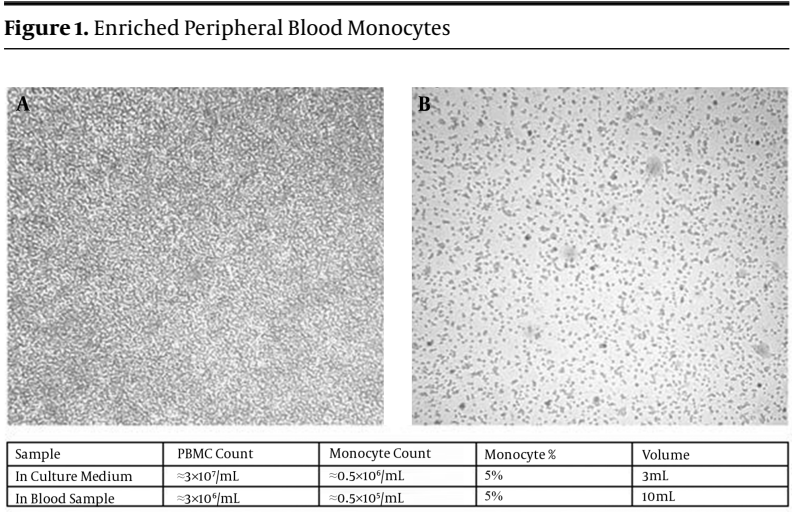

Before (A) and after (B) attachment mediated enrichment process. (Magnification 40x). The table describes cell counts calculated by Trypan blue viable staining method. PBMC: peripheral blood mononuclear cells.

FBS $10 \%$ and $A B$ positive human serum $10 \%$ (both heat inactivated). The process of differentiation was monitored using light microscopy for 14 days. We found that differentiation initiates on day 3 along with spindled morphological changes were observable in media with both supplementations. By the end of day 12, macrophages in both media had differentiated sufficiently, harboring flatted and extended shapes. However, the number of macrophages and viability were higher in media supplemented with $\mathrm{AB}$ positive human serum (Figure 2).

Geimsa staining was used to observe the morphological changes of differentiation in macrophages in details. The macrophages were stained and studied by light microscopy with higher magnification after 12 days of differentiation in $10 \%$ human $\mathrm{AB}$ positive human serum. A normal and distinct feature of macrophage morphology was observed including increased cytoplasmic ratio, pseudopodia and vacuolar system indicating phagocytic activity(Figure 3). Interestingly, all macrophages displayed similar phenotypic characteristics and were morphologically homogenous.

Phagocytosis is the most important function of macrophages as a scavenger cell. We were interested to study if the resulted differentiated macrophages were able to accomplish the phagocytosis process successfully. Non-opsonized SYBR stained heat inactivated E. coli was used for phagocytosis assay according the method described previously. With fluorescent microscopy imaging, we noticed not all but a number of macrophages engulfed green colored bacteria successfully during phagocytosis assay (Figure 4).
Figure 2. Macrophages Differentiation Monitoring by Light Microscopy
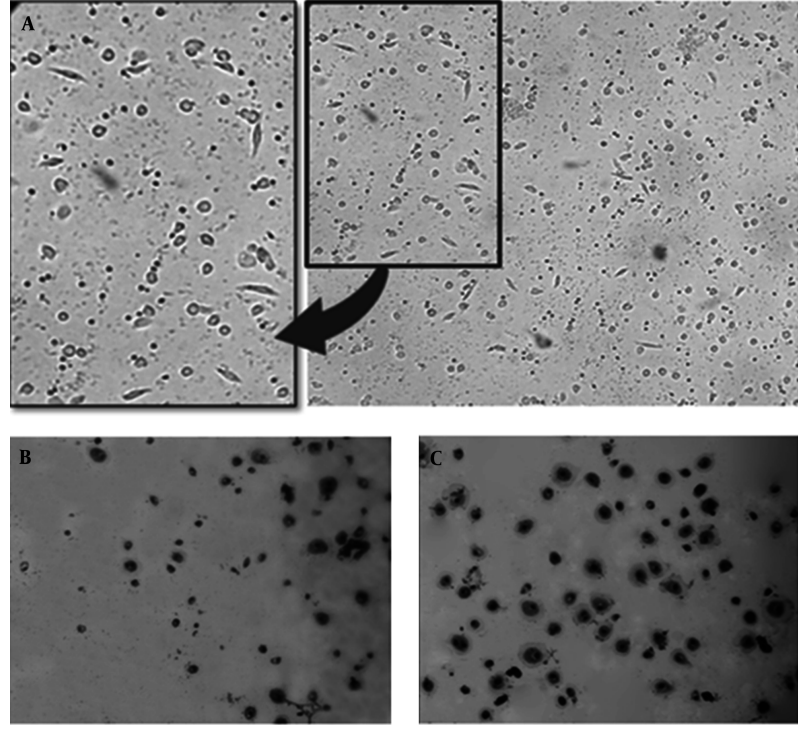

Early at day 3 the spindled shape macrophages were observed although non differentiated monocytes were noticeable (A). As shown by Geimsa staining, by the end of day 12, almost all of cells were differentiated to macrophage morphology (B and $C$ ), although culture in human $\mathrm{AB}$ positive serum (C) provided more better results (B). (Magnification 40x)

Figure 3. Distinct Morphology of Differentiated Macrophages Stained by Geimsa
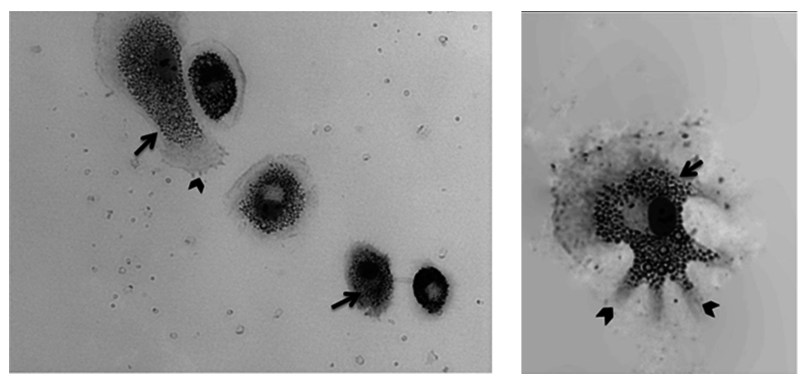

The increased cytoplasmic ratio, pseudopodia (arrow head) and extended vacuolar system (arrow) are shown. (Magnification 400x)

\section{Discussion}

Here, we presented an optimized method to culture and differentiate PBMCs into MDMs and obtaining functional macrophages which are able to function and phagocyte efficiently. Culture and differentiation of monocyte derived macrophages can provide an applicatory in vitro tool for studies related to innate immunity and inflammatory diseases [18].

Monocytes constitute about $2 \%-8 \%$ of WBC population in peripheral blood [3]. Utilizing specific surface markers in magnetic activated cell sorting (MACS) selection to 

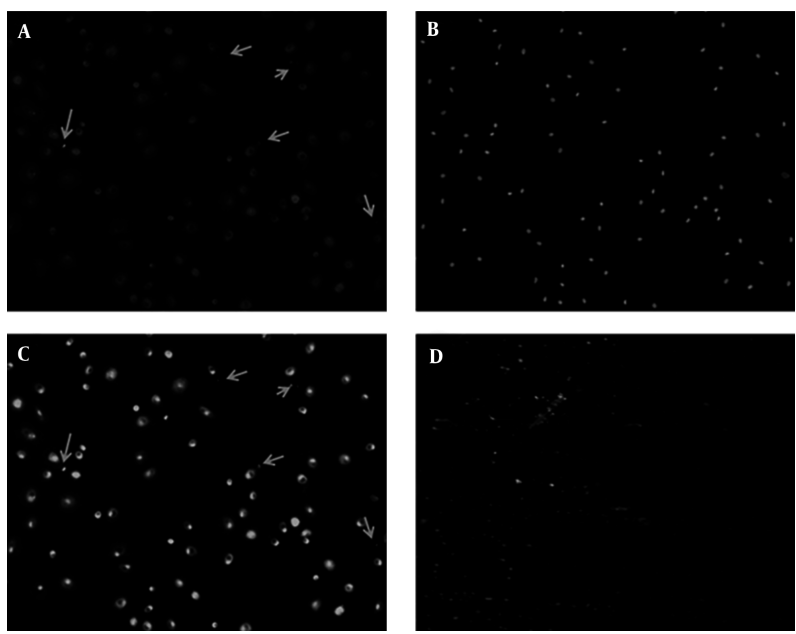

Figure 4. Phagocytosis assay, macrophages engulfed green colored bacteria (marked by Arrows) successfully (A), B shows blue stained nucleus of same section with DAPI. Merged A and B indicates cytoplasmic position of green colored phagocytes (C). The heat inactivated SYBR stained E. coli is imaged by fluorescent microscopy as a control (D). (Magnification 100x)

purify monocytes from PBMC is feasible but this requires more facilities, indeed a remarkable proportion of monocytes might be missed due to restriction factors such as antigen-antibody cross reaction [6]. According to literature, monocyte purification based on their distinctive affinity of attachment can yield $95 \%$ purity $[8,15]$. Some researchers have reported gelatin coating or fibronectin coating [19] and plasma treatment the surface of culture plates can facilitate the attachment [12] while we found no difference between monocyte attachment enrichment in gelatin or plasma treated and non-treated tissue culture plates.

Differentiation of macrophages is a regulated process depending on conditions which can result in classical M1 or alternative M2 macrophages [4]. Different populations of macrophages can exhibit different features and functions [3]. While G-CSF enhances M1 differentiation, GMCSF promotes M2 phenotypes [20]. Some researchers supply both GCSF and GMCSF to avoid this bias, but it actually disturbs the plasticity of macrophages differentiation [21, 22]. This method is unprofitable when the differentiation plasticity or M1/M2 ratio is the main subject of study. Some older protocols have used human serum instead for macrophages differentiation [11], although less macrophage proliferation will occur in this case. Here, we showed $A B$ positive blood type human serum is the best choice for this purpose, since it is preferred by human cells in comparison to FBS and indeed has no cross reaction with probable RBCs contamination. The resulted macrophages present typical morphology with increased cytoplasmic ratio, pseudopodia and vacuolar system.

In conclusion, this optimized method is a simple and cost effective method to prepare monocyte derived macrophages with typical morphology representations able to phagocytosis efficiently; applicable for in vitro functional studies of macrophages.

\section{Acknowledgments}

This article is extracted from a research study financially supported by Golestan University of Medical Sciences (grant number: 930618118). The authors also would like to thank personnel of cell culture lab and molecular medicine lab in faculty of advanced medical technologies specially Mrs. M. Haydari, Dr S. Zhand and Dr Ayoob Khosravi for being great supports.

\section{Footnotes}

Authors' Contribution: All authors are contributed equally to this work.

Conflict of Interest: The authors declare that there is no conflict of interest regarding the publication of this article.

Funding/Support: Golestan University of Medical Sciences.

\section{References}

1. Martinez FO, Sica A, Mantovani A, Locati M. Macrophage activation and polarization. Front Biosci. 2008;13:453-61. [PubMed: 17981560].

2. Wynn TA, Chawla A, Pollard JW. Macrophage biology in development, homeostasis and disease. Nature. 2013;496(7446):445-55. doi: 10.1038/nature12034. [PubMed: 23619691].

3. Gordon S, Taylor PR. Monocyte and macrophage heterogeneity. Nat Rev Immunol. 2005;5(12):953-64. doi: 10.1038/nri1733. [PubMed: 16322748].

4. Rey-Giraud F, Hafner M, Ries $\mathrm{CH}$. In vitro generation of monocytederived macrophages under serum-free conditions improves their tumor promoting functions. PLoS One. 2012;7(8):e42656. doi: 10.1371/journal.pone.0042656. [PubMed: 22880072].

5. Daigneault M, Preston JA, Marriott HM, Whyte MK, Dockrell DH. The identification of markers of macrophage differentiation in PMAstimulated THP-1 cells and monocyte-derived macrophages. PLoS One. 2010;5(1):e8668. doi: 10.1371/journal.pone.0008668. [PubMed: 20084270].

6. Menck K, Behme D, Pantke M, Reiling N, Binder C, Pukrop T, et al. Isolation of human monocytes by double gradient centrifugation and their differentiation to macrophages in teflon-coated cell culture bags. JVis Exp. 2014(91):e51554. doi: 10.3791/51554. [PubMed: 25226391].

7. Zizzo G, Hilliard BA, Monestier M, Cohen PL. Efficient clearance of early apoptotic cells by human macrophages requires M2c polarization and MerTK induction. J Immunol. 2012;189(7):3508-20. doi: 10.4049/jimmunol.1200662. [PubMed: 22942426].

8. Czuprynski CJ, Hamilton $\mathrm{H}$. The effects of serum on the in vitro adherence and maturation of bovine monocytes. Vet Immunol Immunopathol. 1985;9(2):189-93. [PubMed: 4035976]. 
9. Freundlich B, Avdalovic N. Use of gelatin/plasma coated flasks for isolating human peripheral blood monocytes. J Immunol Methods. 1983;62(1):31-7. [PubMed: 6875265].

10. Vissers MC, Jester SA, Fantone JC. Rapid purification of human peripheral blood monocytes by centrifugation through Ficoll-Hypaque and Sepracell-MN. J Immunol Methods. 1988;110(2):203-7. [PubMed: 2837516].

11. Musson RA. Human serum induces maturation of human monocytes in vitro. Changes in cytolytic activity, intracellular lysosomal enzymes, and nonspecific esterase activity. Am J Pathol. 1983;111(3):33140. [PubMed: 6859218].

12. Proctor RA, Textor JA, Vann JM, Mosher DF. Role of fibronectin in human monocyte and macrophage bactericidal activity. Infect Immun. 1985;47(3):629-37. [PubMed: 3972444].

13. Rees AJ. Monocyte and macrophage biology: an overview. Semin Nephrol. 2010;30(3):216-33. doi: 10.1016/j.semnephrol.2010.03.002. [PubMed: 20620668].

14. de Mulder PH, van Rennes H, Mier PD, Bergers M, de Pauw BE, Haanen C. Characterization of monocyte maturation in adherent and suspension cultures and its application to study monocyte differentiation in Hodgkin's disease. Clin Exp Immunol. 1983;54(3):681-8. [PubMed 6360444].

15. Wahl LM, Wahl SM, Smythies LE, Smith PD. Isolation of human monocyte populations. Curr Protoc Immunol. 2006;Chapter 7:Unit 7 6A. doi: 10.1002/0471142735.im0706as70. [PubMed: 18432977].
16. de Almeida MC, Silva AC, Barral A, Barral Netto M. A simple method for human peripheral blood monocyte isolation. Mem Inst Oswaldo Cruz. 2000;95(2):221-3. [PubMed:10733742]

17. Fleetwood AJ, Dinh H, Cook AD, Hertzog PJ, Hamilton JA. GM-CSF- and M-CSF-dependent macrophage phenotypes display differential dependence on type I interferon signaling. J Leukoc Biol. 2009;86(2):41121. doi: 10.1189/jlb.1108702. [PubMed: 19406830].

18. Ma J, Chen T, Mandelin J, Ceponis A, Miller NE, Hukkanen M, et al. Regulation of macrophage activation. Cell Mol Life Sci. 2003;60(11):233446. doi: 10.1007/s00018-003-3020-0. [PubMed:14625680].

19. Perri RT, Kay NE, McCarthy J, Vessella RL, Jacob HS, Furcht LT. Fibronectin enhances in vitro monocyte-macrophage-mediated tumoricidal activity. Blood. 1982;60(2):430-5. [PubMed: 7093527].

20. Hamilton JA. Colony-stimulating factors in inflammation and autoimmunity. Nat Rev Immunol. 2008;8(7):533-44. doi:10.1038/nri2356. [PubMed: 18551128].

21. Martinez FO, Gordon S. The M1 and M2 paradigm of macrophage activation: time for reassessment. F1000Prime Rep. 2014;6:13. doi: 10.12703/P6-13. [PubMed: 24669294].

22. Jaguin M, Houlbert N, Fardel O, Lecureur V. Polarization profiles of human M-CSF-generated macrophages and comparison of M1-markers in classically activated macrophages from GM-CSF and M-CSF origin. Cell Immunol. 2013;281(1):51-61. doi: 10.1016/j.cellimm.2013.01.010. [PubMed: 23454681]. 